\title{
Antimicrobial or Antibacterial coatings? Do they matter!
}

\author{
Atul Tiwari ${ }^{1}$ and Natarajan Ganesan ${ }^{1}$ \\ ${ }^{1}$ Flora Coatings, 275 N Gateway Dr, Phoenix, AZ 85034
}

July 27, 2021

\section{Introduction}

The Covid-19 global pandemic has made the world become increasingly conscious of infectious microorganisms, and their effect on public health. Suddenly, everyone is conscious of the surfaces they touch or encounter, the crowds they get into, and much more.

Numerous products, including protective coatings, offer different types of protection, and the standard scientific terms used in those products often look interchangeably similar. For instance, many people may feel that the terms antibacterial and antimicrobial represent the same thing.

It is in this context that the use of terms such as antibacterial and antimicrobial, besides others, need to be clarified and placed in context. The use of these terms has typically been in reference to antibiotics and medicines; it has been less so with respect to products that prevent infections, using protective surfaces; with the exception being disinfectants and cleaning products. Even therein, the protection offered is often transient and requires regular use of these products. There have been some pioneering approaches in this regard wherein the development of anti-fouling chemistries for uses elsewhere was envisioned to be applicable on public surfaces ${ }^{1}$. It is only of late, especially with the onset of the Covid-19 pandemic, that thoughts and approaches are being focused on developing smart antimicrobial coatings ${ }^{2}$.

Keeping this in mind the following write-up revisits the basics of Microbiology and places them in context with products being marketed. Some bit of background information is also worth looking into the nature and classification of products offering protection against infectious microorganisms.

\section{What does antibacterial mean?}

In simple terms anything that acts against bacteria is antibacterial. Bacteria are a subset of the world of microorganisms (microbes, in short) that surround us. These include viruses, algae, fungi, protists, among others. Among these pathogenic bacteria cause infections and diseases when they enter the host, which could be a plant or animal. These bacteria, then multiply and manifest themselves as infections in the hosts they have entered into.

Antibacterial agents are typically designed and used for protection against pathogenic and infectious bacteria (not the rest of the microbes). Antibacterial agents are designed to counter the growth of these bacteria. The mechanisms of the actions of these agents may differ BUT they act to counter the growth and spread of bacteria in a body or any surface ${ }^{3}$. 


\section{Classification}

\section{Nature of action}

Antibacterials can be either Bacteriostatic OR Bactericidal. The term bacteriostatic refers to medications whose mechanism of action stalls bacterial cellular activity without directly causing bacterial death ${ }^{4}$. In other words, the former class of agents prevents the growth of bacteria or slows their growth substantially without actually killing them directly. In reality, the demarcation between the two categories is not so sharp and distinct i.e. one that exclusively kills bacteria and another that only inhibits growth ${ }^{5}$. The in vitro microbiological determination is more detailed and elaborate. This classification can be broadly applied to other agents that antifungals, antiparasitic, etc. It is a different matter, though, if there are agents that are exclusive to any of the categories of microorganisms.

\section{Source}

1. Naturally occurring - These are naturally occurring compounds found in either plants or animals. Penicillin is a popular antibiotic that was first found and isolated from the fungus Penicillium. After this Prize-winning discovery by the scientist Alexander Fleming ${ }^{6}$ in 1929, the world of antibiotic therapy paved way for the discovery of more such naturally occurring compounds. Plant-based alkaloids are another vast reservoir of such compounds ${ }^{7}$. The history and the evolution of Tetracyclines follow another such pattern ${ }^{8}$.

2. Semi-Synthetic - These are derivatives of naturally occurring compounds. Again, Penicillin is a classic example that has spawned numerous derivatives and generations of its original nature, ever since its discovery ${ }^{9}$. Another example of such antibiotics are the Macrolide class of antibiotics that are bacteriostatic antibiotics with a broad spectrum of activity against many gram-positive bacteria ${ }^{10}$. Development of resistance to them led to the search for the design of new semi-synthetic macrolide antibiotics $^{11}$.

3. Synthetic - These are purely synthesized using in the labs and are often inspired by naturally occurring compounds that possess similar properties; the difference being knowledge of the pathways unique to microbes are taken into cognizance to make them unique in their mode of action. This approach is believed to offer a new path for the exploitation and improvement of natural products to address the growing crisis in antibiotic resistance ${ }^{12}$.

\section{Range of activity}

When it comes to action against bacterial growth, there are different layers of classification.

Gram staining - the activity is gauged whether they are against Gram-positive AND/OR Gram-negative bacteria ( based on their Gram staining nature - a type of staining procedure to determine the nature of their cell walls).

Spore-forming vs non-spore-forming - This is an important classification since certain pathogens form spores. As a result, their metabolism is different and requires different methods of arresting their growth.

Aerobic vs anaerobic - Based on the nature of the bacteria to grow in an oxygen-rich and deprived environment.

These are just some of the ways of classifying the range of action. Based on such layers of the classification, there are either

1. Broad-spectrum OR

2. Narrow-spectrum antibacterial agents

Each spectrum class has its strengths and weaknesses. Though the activity spectrum of such agents has always referred to antibiotics or medicinal compounds, protective coatings have not been often looked at from such an angle. Only a few coatings like INVESIL have been investigated as such ${ }^{13}$. 


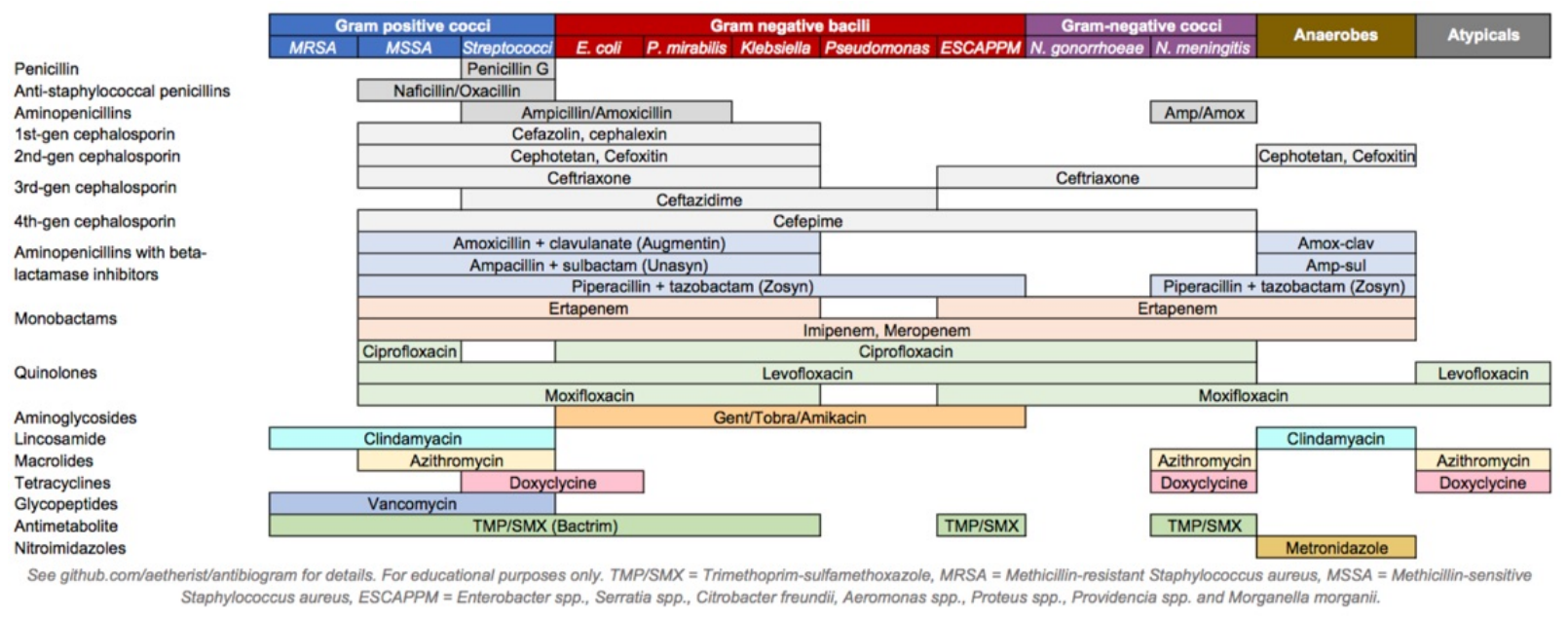

Figure 1: Source: By Akinesia - Own work, CC BY-SA 4.0, https://commons.wikimedia.org/w/index. php? curid $=64751804$

\section{Chemical Classes}

There are different chemical classes of compounds that comprise the antibacterials and these include

1. Beta Lactams

2. Aminoglycosides

3. Quinolones and Fluoroquinolones

4. Streptogramins

5. Sulfonamides

6. Tetracyclines

7. Nitroimidazoles

The list is by no means comprehensive or complete. Suffice to say the list keeps expanding as more and more classes are being introduced ${ }^{14}$.

\section{Cellular functions as targets}

Antibacterial agent-mediated cell death is a complex process that begins with the physical interaction between a drug molecule and its bacterial-specific target and involves alterations to the affected bacterium at the biochemical, molecular, and ultrastructural levels ${ }^{15}$. Hence, antibacterial agents can also be classified based on the targets of action in the bacterial cells or other cells among other microbes. Since microbes vary from being prokaryotes (simple unicellular organisms without a dedicated nucleus) to advanced eukaryotes (mostly unicellular microbes with advanced cellular architecture), the targets of action vary. The more advanced they are the more they resemble the cellular machinery of the humans, thereby making it difficult to achieve exclusive antimicrobial properties. Some of these include

1. Cell wall synthesis inhibitors

2. Membrane function inhibitors

3. Protein Synthesis inhibitors

4. Nucleic acid synthesis inhibitors 


\section{Antimicrobials}

Antimicrobial is a broad term encompassing all agents that act against all types of microorganisms. The term antimicrobial is derived by combining three Greek words, 'anti,' which means 'against, 'mikros' which means 'little,' and 'bios,' which means 'life'.

Herein the agents are more than just antibiotics. They also include agents for use on external surfaces of objects of daily use. Coatings form an important category here ${ }^{16}$. The classes of antimicrobials include

$$
\begin{array}{ll}
* & \text { Antibacterial against bacteria } \\
* & \text { Antifungal against fungi } \\
* & \text { Antiviral against virus } \\
* & \text { Antiparasitic against parasites } \\
* & \text { Antiprotozoal against protozoa } \\
* & \text { Broad-spectrum therapeutic deal with a wide range of microbes }
\end{array}
$$

* Non-pharmaceutical antimicrobials are natural, and some non-medicinal chemical compounds to kill microbes include essential oils, organic acids, antimicrobial pesticides, antimicrobial metals, and alloys.

Antimicrobial scrubs that do not allow the growth of stains and odours on scrubs used for cleaning

* $\quad$ Ozone destroys microbes in water, air, and process equipment.

* Physical processes like Heat sterilization and Radiation are also used to create an antimicrobial effect.

However, the main category of antimicrobials includes ${ }^{17}$ :

* Disinfectants that destroy numerous microbes on non-living surfaces to prevent the spread of infections and diseases

* Antiseptics that are applied over living tissue to prevent infections

* Antibiotics that protect against microbial attack inside the body

Antimicrobials can be microbicidal and biostatic. Like the earlier definition for antibacterial agents, microbicidal agents are those that destroy microbes, while biostatic are the ones that stop their growth.

\section{Antibacterial and Antimicrobial Coatings}

Antibacterial coatings are prepared using three main strategies of contact-killing, antibacterial agent release, and anti-adhesion or bacteria-repelling technology. The antibacterial coating technology is witnessing the growth of new possibilities of multi-functional, multi-release, and multi-approach coatings ${ }^{18}$.

With the advent of numerous harmful microorganisms, some even unknown ones, the coating technology aims for a more versatile smart coating. Multiple new antimicrobial coating technologies are being explored to counter the attack of extremely contagious and harmful pathogens on various surfaces, including plastic, metals, glass, wood, fabric, medicinal implants, surgical equipment, commonly touched surfaces, etc.

Antimicrobial coatings use cellular membrane permeability as the primary weapon against harmful pathogens. The coating compositions include graphene materials (GMs), Graphene-like two-dimensional materials (2DMats), Polycationic hydrogel, polymers, and dendrimers. The latest nanotechnology, mostly silver nanoparticles, is proving its efficacy as a robust antimicrobial agent. 
Today coatings technology is reaching new heights with new developments like smart coatings, self-cleaning coatings, oleophobic coatings, hydrophobic coatings, and numerous other novel coating technologies fulfilling the requirement of multiple surfaces exposed to varied exposure level in different settings.

Apart from protection from harmful pathogens, antimicrobial coatings also greatly reduce maintenance costs while increasing the surface's life span due to being anti-corrosive. With such antimicrobial coatings, the need for harsh cleaning agents is drastically reduced while maintaining health and cleanliness standards. New coating technology also provides a finish to the surface while contributing to the infrastructure standard and conforming to health standards in all settings.

\section{Conclusion}

The terminologies define the scope of the antimicrobial nature of the compound or coating developed; whether it is the -cidal or -static nature of the compound or coating. These in turn help structured understanding and development of different chemistries to develop a variety of products to protect against emerging infections. A clear understanding of the difference between the two terms could help to manufacture better coatings conducive to application. A suitable antibacterial or antimicrobial technology application could lead to numerous advantages like lowering healthcare costs, labor costs, life-cycle cost, along with numerous other beneficial attributes.

\section{References}

1Tiwari DA. Handbook of Antimicrobial Coatings. Elsevier, 2018 DOI:10.1016/c2016-0-01441-9.

2Case Studies - Flora Coatings. https://floracoating.com/company/resources/case-studies/.

3Neu HC, Gootz TD. Antimicrobial Chemotherapy. 1996.

4Loree J, Lappin SL. Bacteriostatic Antibiotics. 2021.

5Pankey GA, Sabath LD. Clinical relevance of bacteriostatic versus bactericidal mechanisms of action in the treatment of Gram-positive bacterial infections.. Clin Infect Dis 2004; 38: 864-70.

6 Fleming A. On the antibacterial action of cultures of a penicillium, with special reference to their use in the isolation of B. influenzae. 1929.. Bull World Health Organ 2001; 79: 780-90.

7Kaefer CM, Milner JA. Herbs and Spices in Cancer Prevention and Treatment. 2011.

8Nelson ML, Levy SB. The history of the tetracyclines.. Ann N Y Acad Sci 2011; 1241: 17-32.

9PENICILLIN DERIVATIVES. http://www.cyto.purdue.edu/cdroms/cyto2/17/chmrx/penems.htm.

10Schönfeld W, Kirst HA, editors. Macrolide Antibiotics. Birkhäuser Basel, 2002 DOI:10.1007/978-3-03488105-0.

11Fernandes P, Martens E, Pereira D. Nature nurtures the design of new semi-synthetic macrolide antibiotics.. J Antibiot (Tokyo) 2017; 70: 527-33.

12Thaker MN, Wright GD. Opportunities for synthetic biology in antibiotics: expanding glycopeptide chemical diversity.. ACS Synth Biol 2015; 4: 195-206.

13Atul Tiwari NG Anupama Chaturvedi. Smart antimicrobial coating with endless applications. TechConnect Briefs 2018; 1: 236-9.

14Overview of Antibiotics - Infections - Merck Manuals Consumer Version. https://www.merckmanuals . com/home/infections/antibiotics/overview-of-antibiotics . 
15Kohanski MA, Dwyer DJ, Collins JJ. How antibiotics kill bacteria: from targets to networks.. Nat Rev Microbiol 2010; 8: 423-35.

16A Guide to Antimicrobial Coatings. https://www.pcimag.com/articles/107649-a-guide-toantimicrobial-coatings? $\mathrm{v}=$ preview .

17Cloutier M, Mantovani D, Rosei F. Antibacterial Coatings: Challenges, Perspectives, and Opportunities.. Trends Biotechnol 2015; 33: 637-52.

18Antimicrobial Coatings - IUPAC - International Union of Pure and Applied Chemistry. https://iupac . org/materialschemistryedu/biomedical/antimicrobial-coatings/ . 